\title{
Fatal Pseudomonas aeruginosa Bronchopneumonia in a Children's Hospital
}

\author{
A. J. BARSON \\ From the Department of Pathology, Hospital for Sick Children, Toronto, Canada
}

\begin{abstract}
Barson, A. J. (1971). Archives of Disease in Childhood, 46, 55. Fatal Pseudomonas aeruginosa bronchopneumonia in a children's hospital. Forty fatal cases of pseudomonas bronchopneumonia were found in a retrospective review of 600 consecutive necropsies in a children's hospital. This type of infection has been much more common in recent years and was shown to have a predilection for the premature infant dying in the neonatal period, who had been mechanically ventilated.
\end{abstract}

Over the past 25 years there has been a progressive increase in the proportion of terminal bronchopneumonic infections attributable to Gram-negative bacteria. This trend was reviewed recently by Pierce et al. (1966). The most common Gramnegative bacteria to be incriminated has been Pseudomonas aeruginosa. Kneeland and Price (1960) reported that this organism was cultured from nearly a quarter of all their patients dying with pneumonia. Their series was concerned with adult patients but there is evidence of a similar trend in children (Asay and Koch, 1960; Cooper, 1967). Pseudomonas infections are an increasing problem in neonatal units, particularly in the premature infant (Laursen, 1962; Barrie, 1965; Bassett, Thompson, and Page, 1965; Arniel et al., 1966; Fierer, Taylor, and Gezon, 1967; Jellard and Churcher, 1967; Monnet, Chassignol, and Vaillaud, 1967; Knights, France, and Harding, 1968). In many of these cases, pseudomonas has been traced to incubators or resuscitation equipment.

Since Fraenkel's description of pseudomonas inflammation in 1917, it has been known that the histopathology produced by this organism is so distinctive as to be diagnostic. It is possible to use this in conjunction with the bacteriology to assess the incidence of pseudomonas infection in necropsy material. The present study is a retrospective review of pseudomonas bronchopneumonia occurring in 600 consecutive necropsies in a large children's hospital, particular attention being paid to the type of patient affected and the relation to mechanical ventilation.

Received 30 June 1970.

\section{Materials and Methods}

The material for this study was based on 600 consecutive necropsies performed in The Hospital for Sick Children, Toronto, during 1967 and 1968. All of these had had the lungs and air passages examined routinely, both by gross inspection and histopathologically. Two groups of patients, which partly overlapped, were differentiated from these.

First, an assessment was made of those children who had bronchopneumonia to a degree sufficient to contribute significantly to their death. The histological sections from the lungs of these cases were reviewed in order to differentiate from the rest those with pathological lesions typical of a pseudomonas infection. Those cases were then correlated with the bacteriology of antemortem blood cultures or postmortem cultures of lung tissue where these were available. Only those patients were accepted as instances of pseudomonas bronchopneumonia where a typical lung pathology was supported by bacteriological culture. In fact, the correlation was very high. Occasionally a positive culture was not supported by the histopathology, presumably because a septicaemia had not established a pneumonia in the tissue sampled by the pathologist or because of postmortem contamination of lung tissue. However, the converse did not occur. When the histopathology was typical of pseudomonas bronchopneumonia the diagnosis was always corroborated by the bacteriology when this had been undertaken.

The second group of patients to be differentiated from the series were those who were recorded at necropsy as having laryngeal or tracheal ulceration characteristic of that resulting from intubation. Insufflation of the cuff of a laryngeal tube is often associated with discrete symmetrical ulcers of the mucosa immediately below each vocal cord. Similarly prolonged tracheal intubation often results in ulceration of the anterior wall of the trachea overlying the forward projection of the tip 


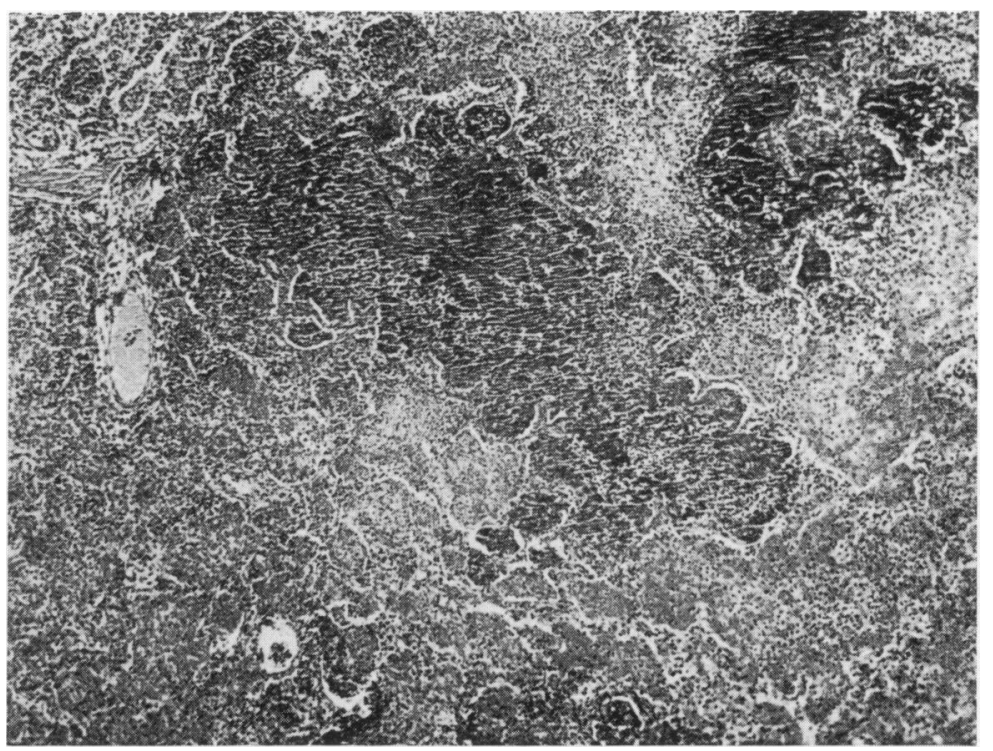

Fig. 1.-Pseudomonas bronchopneumonia in an infant dying at 3 weeks of age. Note the dark areas of necrosis which stain deep blue with haematoxylin. (Haematoxylin and eosin. $\times 60$.)

of a curved tracheotomy or endotracheal tube. An endotracheal cuff may produce a sharply delineated circumferential ulceration of the trachea. The nature and position of the ulcers are so characteristic as to be reliable pathological evidence that the patient was intubated during life, and, by inference, ventilated by external means. This group of patients was correlated with those having terminal bronchopneumonia.

\section{Observations}

Pseudomonas aeruginosa bronchopneumonia is characterized by well-demarcated areas of necrotic consolidation within the lung parenchyma. Under low power magnification these areas of necrosis stain an intense blue with haematoxylin (Fig. 1). Unlike other types of bronchopneumonia, it is essentially a vasculitis, large numbers of bacilli accumulating in the walls of arterioles and venules. So thickly are the vessel walls colonized that they display a distinctive blue haze with a haematoxylin stain or pink with a Gram stain (Fig. 2). The rod-like form of the bacteria is usually readily apparent with higher magnification. This vasculitis itself differs from that seen in other infections in that there is often a completelack of an intraluminal inflammatory response. Peripheral to the vessel there is usually a surprisingly mild inflammatory cell infiltration in which lymphocytes and mononuclear cells predominate together with an extensive necrosis. At a later stage, the vessel wall is disrupted (Fig. 3), and haemorrhage into adjacent alveoli is a commonly associated feature.

On the basis of this histopathology, substantiated by bacteriological culture, $\mathbf{4 0}$ cases of Pseudomonas aeruginosa bronchopneumonia were found in 600 consecutive necropsies in 1967 and eight months of 1968. A comparison with the incidence of staphylococcal bronchopneumonia in necropsies performed in the same hospital shows how this has declined in importance since the 1950's. The incidence of terminal pseudomonas lung infection since the beginning of 1967 was comparable to that formerly seen with staphylococcus (Table I).

TABLE I

Incidence of Staphylococcal and Pseudomonas Bronchopneumonia in Necropsies at the Hospital for Sick Children from 1955 to 1968

\begin{tabular}{l|c|c|c|c|c|c|c|c|c|c|c|c|c|c}
\hline \multicolumn{1}{c|}{ Year } & 55 & 56 & 57 & 58 & 59 & 60 & 61 & 62 & 63 & 64 & 65 & 66 & 67 & $68 \star$ \\
\hline $\begin{array}{l}\text { Staphylococcus } \\
\text { Pseudomonas }\end{array}$ & 68 & 33 & 12 & 16 & 8 & 7 & 8 & - & 7 & 1 & 2 & $\begin{array}{r}1 \\
1\end{array}$ & $\begin{array}{r}1 \\
30\end{array}$ & $\begin{array}{r}1 \\
10\end{array}$ \\
\hline
\end{tabular}

*January to August only. 


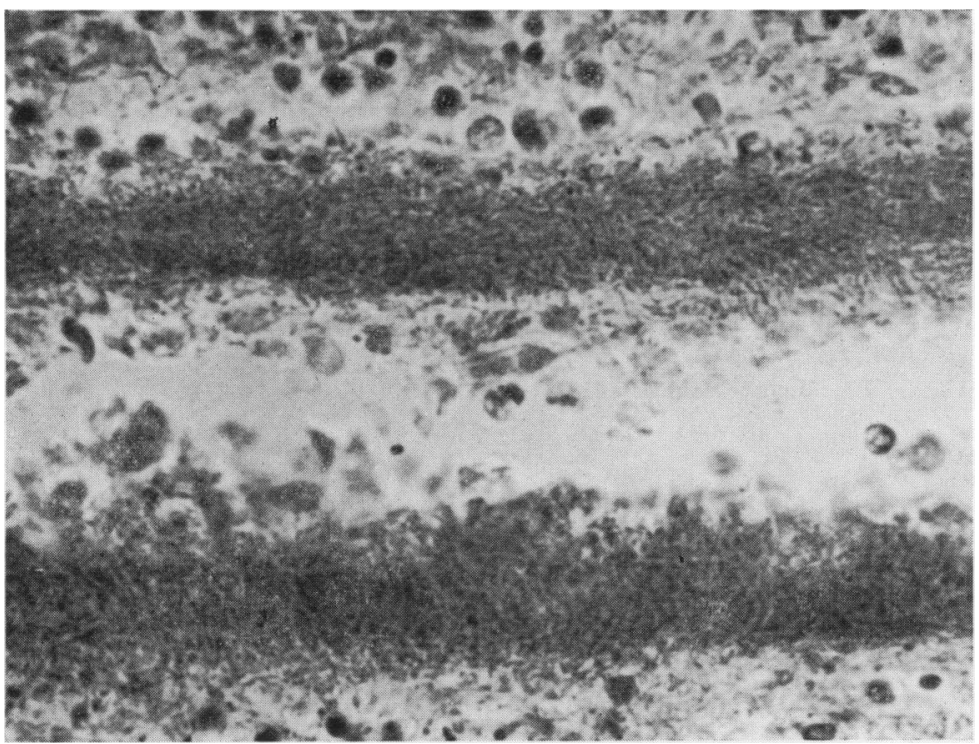

FIG. 2.-An arteriole cut longitudinally to show the dense bacterial colonization of the wall. There is no intraluminal pathology but peripherally there are a few mononuclear inflammatory cells. (Gram. $\times 120$.)

Altogether $176(29 \%)$ of the necropsies had a complicating terminal pneumonia, and pseudomonas infection accounted for just over a fifth of them. The relation of these cases to intubation and laryngeal or tracheal ulceration is shown in Table II.
Nearly one-third of all the necropsies (193) had airway mucosal ulceration evident at necropsy. Of these, nearly half (93) had a bronchopneumonia.

The clinical histories of all the children with pneumonia were reviewed to assess the relation of

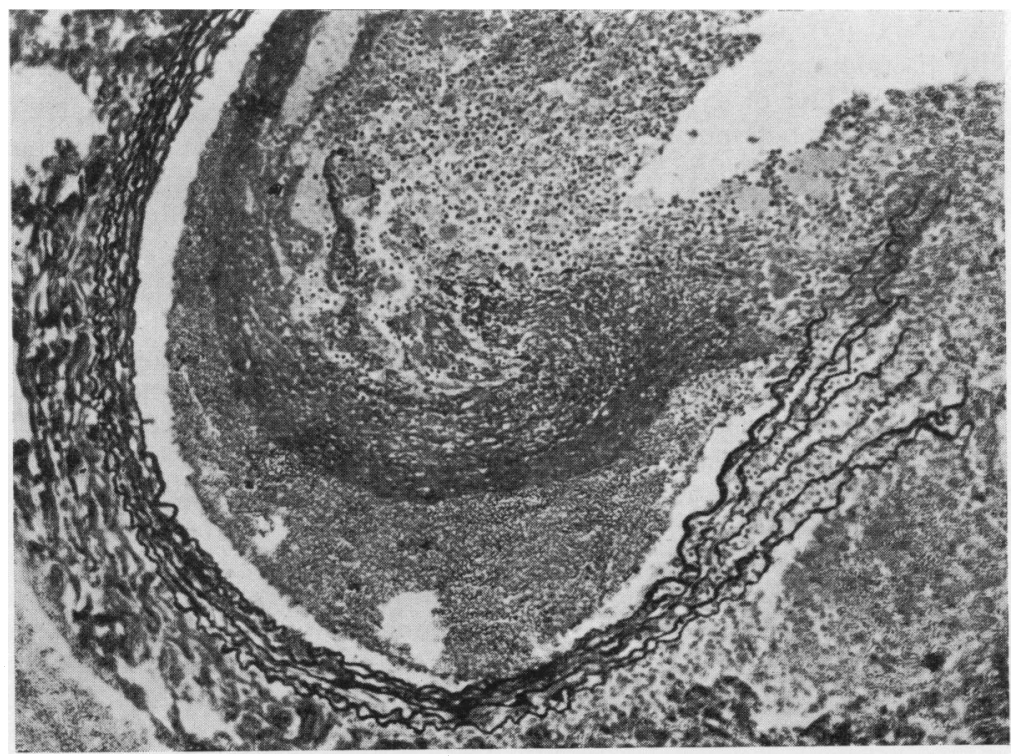

Fig. 3.-A pulmonary arteriole ruptured by pseudomonas inflammation, with haemorrhage into the adjacent alveolae. The lumen contains thrombus. The elastic fibres in the wall of the vessel on the right are splayed and fragmented. (Elastic van Gieson. $\quad \times 90$.) 


\section{TABLE II}

Analysis of 600 Consecutive Necropsies in 1967-8 According to Presence and Type of Bronchopneumonia and Occurrence of Laryngeal or Tracheal Ulceration

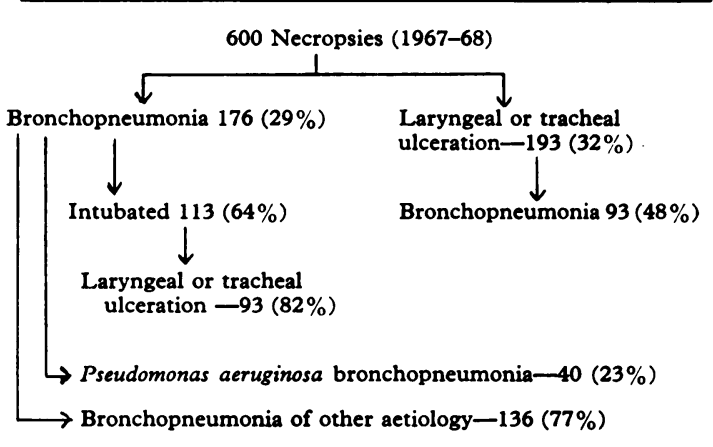

Percentages in brackets are those within immediately preceding category.

intubation to ulceration. It was found that the majority of the cases with terminal bronchopneumonia had been intubated during their last illness (113 out of 176) and that 93 (82\%) of these had had airway ulceration observed after death.

A comparison is made in Table III between the incidence of intubation and of ulceration in children with pseudomonas bronchopneumonia and with pneumonias due to other organisms. Applying the $\chi^{2}$ test both intubation $(0.01<\mathrm{P}<0.025)$ and ulceration $(0.025<\mathrm{P}<0.05)$ were significantly more frequent with pseudomonas infections compared with other bacteria. Out of the $\mathbf{4 0}$ children dying with pseudomonas bronchopneumonia, 32 had been artificially ventilated before death. 3 of those were intubated less than 24 hours before death, which is probably too short a time for the ventilator to be incriminated as a source of infection. For 29 children the infection could have been

\section{TABLE III}

Comparison of Pseudomonas Bronchopneumonia and Pneumonias due to Other Organisms with regard to Numbers Intubated and Those Having Laryngeal or Trachael Ulceration at Necropsy

\begin{tabular}{|c|c|c|c|}
\hline & Cases & Intubated & Ulcerated \\
\hline $\begin{array}{l}\text { Pseudomonas broncho- } \\
\text { pneumonias } \\
\text { Other bronchopneumonias }\end{array}$ & $\begin{array}{r}40 \\
136\end{array}$ & $\begin{array}{l}32(80 \%) \\
81(59.5 \%)\end{array}$ & $\begin{array}{l}27(67 \cdot 5 \%) \\
66(48 \cdot 5 \%)\end{array}$ \\
\hline
\end{tabular}

Note: Significantly more infants with pseudomonas bronchopneumonia were intubated $(0.01<\mathrm{P}<0.025)$ and more were ulcerated $(0.025<P<0.05)$ compared with those dying from other forms of bronchopneumonia. acquired in this way but this is unproved bacteriologically.

Fig. 4 illustrates the relation of pseudomonas and other bronchopneumonias to the

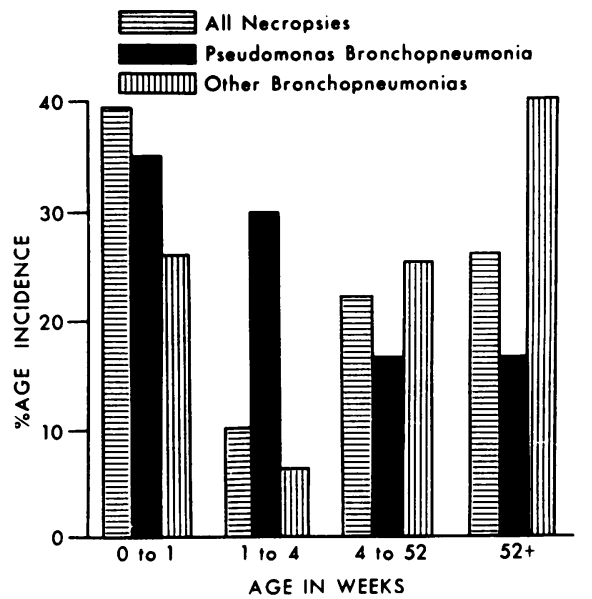

Fig. 4.-A block graph showing the percentage incidence in four age categories of 600 consecutive necropsies compared with the children among these dying with pseudomonas and other forms of bronchopneumonia. Though there were only 40 cases of pseudomonas infection they had a relative predilection for the neonatal period, whereas the other terminal pneumonias (136 cases) were more similar in their age distribution to the total necropsy population.

necropsy population as a whole. The highest death rate in the hospital was among infants in the first week of life, with comparatively few deaths during the remaining 3 weeks of the neonatal period. The incidence of pseudomonas bronchopneumonia was disproportionately high during this period. Pneumonias caused by bacteria other than pseudomonas were relatively more common in children over 1 year of age.

The influence of low birthweight $(2,500 \mathrm{~g}$ or below), on bronchopneumonic deaths in the neonatal period is depicted in Table IV. Using the $\chi^{2}$ test the number of low birthweight infants with pseudomonas bronchopneumonia is significantly higher than in the necropsy population as a whole $(0.01<P<0.025)$ whereas the number of low birthweight neonates dying with other forms of pneumonia is not significantly higher $(0 \cdot 3<P<0 \cdot 4)$.

The infection by pseudomonas was always a complication of another disease. The primary diagnoses in these cases are set out in Table V. The most common of these was respiratory distress syndrome, followed by alimentary tract disorders either obstructive or inflammatory in nature. The 
four cases of congenital heart disease had all undergone recent major surgery.

\section{TABLE IV}

Incidence of Low Birthweight (2,500 $\mathrm{g}$ or less) Among All Neonatal Necropsies and Among Neonatal Deaths with Pseudomonas or Other Forms of Bronchopneumonia

\begin{tabular}{c|c|c|c}
\hline & All Necropsies & $\begin{array}{c}\text { Pseudomonas } \\
\text { Broncho- } \\
\text { pneumonia }\end{array}$ & $\begin{array}{c}\text { Other } \\
\text { Broncho- } \\
\text { pneumonias }\end{array}$ \\
\hline $\begin{array}{c}\text { No. of neonatal } \\
\text { necropsies } \\
\text { Incidence of low } \\
\text { birthweight }\end{array}$ & 293 & 26 & 46 \\
\hline
\end{tabular}

The incidence of low birthweight in those dying of pseudomonas bronchopneumonia is significant $(0.01<P<0.025)$, but is not significant among those dying with other types of bronchopneumonia $(0.3<\mathrm{P}<0.4)$.

\section{TABLE V}

Primary Diagnosis in 40 Infants Dying with Pseudomonas Bronchopneumonia

\begin{tabular}{l|r}
\multicolumn{1}{c|}{ Primary Diagnosis } & No. \\
\hline Respiratory distress syndrome & 16 \\
Alimentary tract obstruction & 5 \\
Congenital heart disease & 4 \\
Leukaemia & 3 \\
Gastroenteritis & 2 \\
Intrauterine asphyxia & 2 \\
Cystic fibrosis & 2 \\
Burns & 1 \\
Alymphoplasia & 1 \\
Tracheo-oesophageal fistula & 1 \\
Meningocele & 1 \\
Adrenal hypoplasia & 1 \\
Diaphragmatic hernia & 1 \\
\end{tabular}

\section{Discussion}

This retrospective study in a children's hospital shows that terminal pseudomonas bronchopneumonia has become common since 1966, assuming in its incidence the place formerly held by staphylococcal infections. It is a peculiarity of pseudomonas aeruginosa, well illustrated by the present study, that it has a predilection for the low birthweight neonatal patient who has been treated by intubation and mechanical ventilation.

In a similar review of adult necropsies using histopathological criteria for diagnosis, Pierce et al. (1966) accounted for the rise in pseudomonas infections after 1963 on the basis of the increase in broad spectrum chemotherapy and contamination of nebulization reservoirs. Tracheotomy was not of itself a predisposing factor, although shock and anaemia were.

At the Hospital for Sick Children, the rise in incidence of pseudomonas bronchopneumonia has followed by some years the routine use of broad spectrum antibiotics, and the phenomenon cannot be wholly explained on this basis. It is the opinion of the author that an important factor has been the increasing use of humidified ventilatory apparatus for debilitated infants, especially in the treatment of respiratory distress syndrome.

Though the observations in the study support such a contention, it is retrospective and no consistent bacteriological studies exist to confirm this. However recent outbreaks at other centres of pseudomonas infection have been traced to incubators (Barrie, 1965; Monnet et al., 1967) and resuscitation equipment (Bassett et al., 1965; Fierer et al., 1967; Phillips, 1967). Moisture is an essential factor in the epidemiology of pseudomonas infections, airborne outbreaks being uncommon. The organism has a low pathogenicity for healthy people, and a carrier state as high as $12 \%$ has been reported (British Medical Journal, 1967). It has been shown in both children and adults undergoing major surgery with endotracheal intubation that the upper respiratory tract may become rapidly colonized by Gram-negative organisms from the patient himself rather than from the hospital environment (Redman and Lockey, 1967).

Eleven of the patients in the present study had undergone surgery before infection, 5 of whom had had alimentary tract obstruction. An association with surgery for gastrointestinal defects has been noted before (Asay and Koch, 1960; Cooper, 1967) and could be explained by a carrier state (Jellard and Churcher, 1967).

Nevertheless, it is likely that a high proportion of the cases was the result of cross-infection rather than self-colonization. Humidifying equipment is the most common reservoir of pseudomonas infection in the neonatal unit, and the difficulties in keeping this sterile cannot be stressed too much (Laursen, 1962).

Related to this problem is the fact that $82 \%$ of the children in this series with terminal bronchopneumonia who had been intubated had developed mucosal ulceration of the larynx or trachea. Such ulceration produced by an indwelling tube can easily become infected (Teplitz et al., 1964) by direct inoculation with droplet borne pseudomonas. Inhalation of material from the infected ulcer may then result in a fatal pneumonia.

The proneness of the neonate to pseudomonas infection in the present study is in agreement with that found by Asay and Koch (1960) at another 
centre. This is probably a reflection of a lowered immunological capacity in early postnatal life. Similarly, when this capacity is decreased by disease, such as leukaemia, or immunosuppressive drugs such as the corticosteroids, there is also a predisposition to pseudomonas infection (British Medical Journal, 1967). Among the neonatal infants in the present series, the low birthweight infant was particularly at risk. This is in part a consequence of its immunological immaturity, and in part a consequence of treatment by artificial ventilation and humidification with broad spectrum antibiotic cover. Surgery and steroid therapy were additional factors in some cases.

It is likely that the active treatment of premature infants and the use of ventilatory apparatus will increase in the future. Unless the most stringent bacteriological supervision is employed, the incidence of pseudomonas infection in the children's hospital is unlikely to diminish. Radiography may also provide a valuable aid to diagnosis (Joffe, 1969).

Pathologically pseudomonas infection is distinct in that bacillary invasion appears to originate within capillaries and subsequently invade larger blood vessels from the outer layers towards the lumen (Teplitz, 1965). Disruption of arteriolar walls (Fig. 3) with haemorrhage is a common feature, and it is interesting that two elastases have been recently isolated from cultures of the organism (Suss et al., 1969). This vasculitis is sufficiently characteristic histologically for it to be worth noting at necropsy, even in the absence of bacteriological confirmation. It is important to use this peculiarity to assess the future trends in the incidence of pseudomonas infection.

I wish to thank Dr. M. J. Lynch and Dr. A. J. Wort for their advice. The work was supported by grants from the Wellcome Trust and the National Fund for Research into Crippling Diseases.

\section{REFERENCES}

Arniel, C., Fleurquin, N., Larroche, J. C., and Minkowski, A. (1966). A propos de 18 observations d'infections à "Pseudomonas" du nouveau-né. Considerations epidemiologiques. Archives Françaises de Pédiatrie, 23, 413.
Asay, L. D., and Koch, R. (1960). Pseudomonas infections in infants and children. New England fournal of Medicine, 262, 1062.

Barrie, D. (1965). Incubator-borne Pseudomonas pyocyanea infection in a newborn nursery. Archives of Disease in Childhood, 40, 555.

Bassett, D. C. J., Thompson, S. A. S., and Page, B. (1965). Neonatal infections with Pseudomonas aeruginosa, associated with contaminated resuscitation equipment. Lancet, $1,781$.

British Medical fournal (1967). Pseudomonas infection in hospital. (Leading article). 4, 309.

Cooper, R. G. (1967). Systemic Pseudomonas infection in childhood. Medical fournal of Australia, 1, 527.

Fierer, J., Taylor, P. M., and Gezon, H. M. (1967). Pseudomonas aeruginosa epidemic traced to delivery-room resuscitators. New England Fournal of Medicine, $276,991$.

Fraenkel, E. (1917). Weitere Untersuchungen über die Menschenpathogenităt des Bacillus pyocyaneus. Zeitschrift für Hygiene und Infektionskrankheiten, 84, 369.

Jellard, C. H., and Churcher, G. M. (1967). An outbreak of Pseudomonas aeruginosa (pyocyanea) infection in a premature baby unit, with observations on the intestinal carriage of Pseudomonas aeruginosa in the newborn. Fournal of Hygiene, 65, 219.

Joffe, N. (1969). Roentgenologic aspects of primary Pseudomonas aeruginosa pneumonia in mechanically ventilated patients. American fournal of Roentgenology, 107, 305.

Kneeland, Y., Jr., and Price, K. M. (1960). Antibiotics and terminal pneumonia: a post-mortem microbiological study. American Fournal of Medicine, 29, 967.

Knights, H. T., France, D. R., and Harding, S. (1968). Pseudomonas aeruginosa cross infection in a neonatal unit. New Zealand Medical fournal, 67, 617.

Laursen, H. (1962). Incidence of Pseudomonas aeruginosa and other gram-negative rods in newborn infants. Acta Obstetricia et Gynecologica Scandinavica, 41, 254.

Monnet, P., Chassignol, S., and Vaillaud, J. C. (1967). Infections à 'Pseudomonas aeruginosa' dans un centre de prématures durant les années 1960 à 1963. Archives Françaises de Pédiatrie, 24, 543.

Phillips, I. (1967). Pseudomonas aeruginosa respiratory tract infections in patients receiving mechanical ventilation. fournal of Hygiene, 65, 229.

Pierce, A. K., Edmonson, E. B., McGee, G., Ketchersid, J., Loudon, R. G., and Sanford, J. P. (1966). An analysis of factors predisposing to gram-negative bacillary necrotizing pneumonia. American Review of Respiratory Disease, 84, 309.

Redman, L. R., and Lockey, E. (1967). Colonisation of the upper respiratory tract with gram-negative bacilli after operation, endotracheal intubation and prophylactic antibiotic therapy. Anaesthesia, 22, 220.

Suss, R. H., Fenton, J. W., II, Muraschi, T. F., and Miller, K. D. (1969). Two distinct elastases from different strains of Pseudomonas aeruginosa. Biochimica et Biophysica Acta, 191, 179.

Teplitz, C. (1965). Pathogenesis of Pseudomonas vasculitis and septic lesions. Archives of Pathology, 80, 297.

-, Epstein, B. S., Rose, L. R., and Moncrief, J. A. (1964). Necrotizing tracheitis induced by tracheostomy tube: pathogenesis. Archives of Pathology, 77, 6.

Correspondence should be addressed to Dr. A. J. Barson, Department of Pathology, University of Manchester, Manchester, M13 9PL. 